\title{
INNERVATION OF AN AMPHIPOD HEART
}

\author{
By J. S. Alexandrowicz \\ The Plymouth Laboratory
}

(Text-figs. I and 2)

For investigation of the innervation of the heart in the Amphipoda, Marinogammarus ${ }^{\star}$ marinus has been chosen as it is among the largest species available at Plymouth. The smaller size of other species added greatly to the difficulties in making the preparations. Some observations were made with Gammarus locusta, but because of certain features of its anatomy referred to below it was found to be less suitable for this kind of study.

For staining of the nerves methylene blue was used. The immersion of the tissues into a weak solution of this dye (IO-15 drops of $0.5 \%$ solution in distilled water to $100 \mathrm{ml}$. of sea water) gave more constant results than the injection of a stronger solution into the body of the animal. However, the staining of certain elements, such as the nerve cells in the ganglionic trunk, could be obtained with the latter method only. The individual reactions of the animals to the staining varies greatly: thus, when several specimens are injected with the same solution at the same time the nerves of some stain more or less well while for others the results are poor or often nil.

To expose the heart the ventral body wall of the animal should be cut along the middle line or, to avoid damage to the ventral neural cord, along the side of the latter. After the parts of the sectioned body wall have been pulled out and pinned to the paraffin plate, the digestive organs are removed, leaving in situ the two dorsal diverticula which are so linked with the posterior aorta that it is practically impossible to separate them from it. The gonads should also be removed. The digestive organs are connected with the pericardial diaphragm by strands of connective tissue, but there is no particular difficulty in severing these connexions and in obtaining preparations with the heart tube and the pericardial diaphragm in their normal positions. In G. locusta, however, this diaphragm seems to be a little weaker and is more easily damaged; the heart tube then becomes flaccid or even displaced, and subsequent manipulations are much more difficult.

Such preparations with the heart and the pericardium left in situ show many of the details described below, but for better observation of heart nerves the heart tube should be opened along its middle line. This operation can be done with a very fine scalpel and preferably after some staining has

* Separated from Gammarus by Sexton \& Spooner (I940). 
made the outlines of the tissue elements more distinguishable. After the heart has been opened its ganglionic trunk is more easily accessible for staining and observation, but even then, being situated on the outside of the dorsal heart wall, it is seen by transparency only. For its direct observation the heart has to be isolated and mounted with the dorsal side up. This is a difficult operation, and it is more expedient to perform it at one of the later stages, after the preparations have been stained and fixed and are already either in water or alcohol or xylol.

The fixation of the staining has not been quite satisfactory with the tissue of Marinogammarus. As is already known, the elements stained with methylene blue, to be well fixed with ammonium molybdate solution, must attain a certain intensity of colour. In gammarids some of the nerves do not reach this point, and although they show up distinctly before being fixed they turn pale or even become indistinguishable in the completed preparations. Neither was the double fixation, i.e. in ammonium picrate followed by ammonium molybdate, of much help. With such material the best practice is, after having examined the permanent preparations, to check repeatedly the dubious points on fresh preparations during the staining process before their fixation.

\section{The Anatomy of the Heart}

The heart of Marinogammarus has the shape of a tube extending from the posterior edge of the first thoracic segment (which enters into the composition of the cephalothorax) to the middle of the 7 th thoracic segment. Its wall is made up of a layer of cross-striated muscle fibres turning in left-handed spirals surrounded by connective tissue fibres of longitudinal direction. Numerous rounded cells adhere to the inside of the muscle fibres without forming a continuous layer. The three pairs of ostial orifices piercing the lateral heart wall are situated in the $3 \mathrm{rd}$, $4^{\text {th }}$ and 5 th thoracic segments. In front and backwards the heart-tube continues with the anterior and posterior aorta respectively (Fig. IA).

Four pairs of arteries arise from the heart: the first, running in anterolateral direction, starts near the origin of the anterior aorta, while three others originate on the ventral side of the heart in the 4 th, 5 th and 6 th segments. The walls of the arteries are very thin and not often noticeable, but their valves sometimes stain more deeply than the muscles of the heart, and then stand out distinctly. Even the play of these valves opening at each systole can occasionally be seen. Klövekorn (I934), who found these vessels in Gammarus pulex, called them 'Darm-Leber-Arterien', since they run to the digestive organs. It can be added that in methylene-blue preparations some finer branches of these vessels can be seen to ramify in the pericardial septum.

The heart is suspended in the pericardial cavity by means of bands of connective tissue, the strongest of them stretching from the terga to the dorsal 


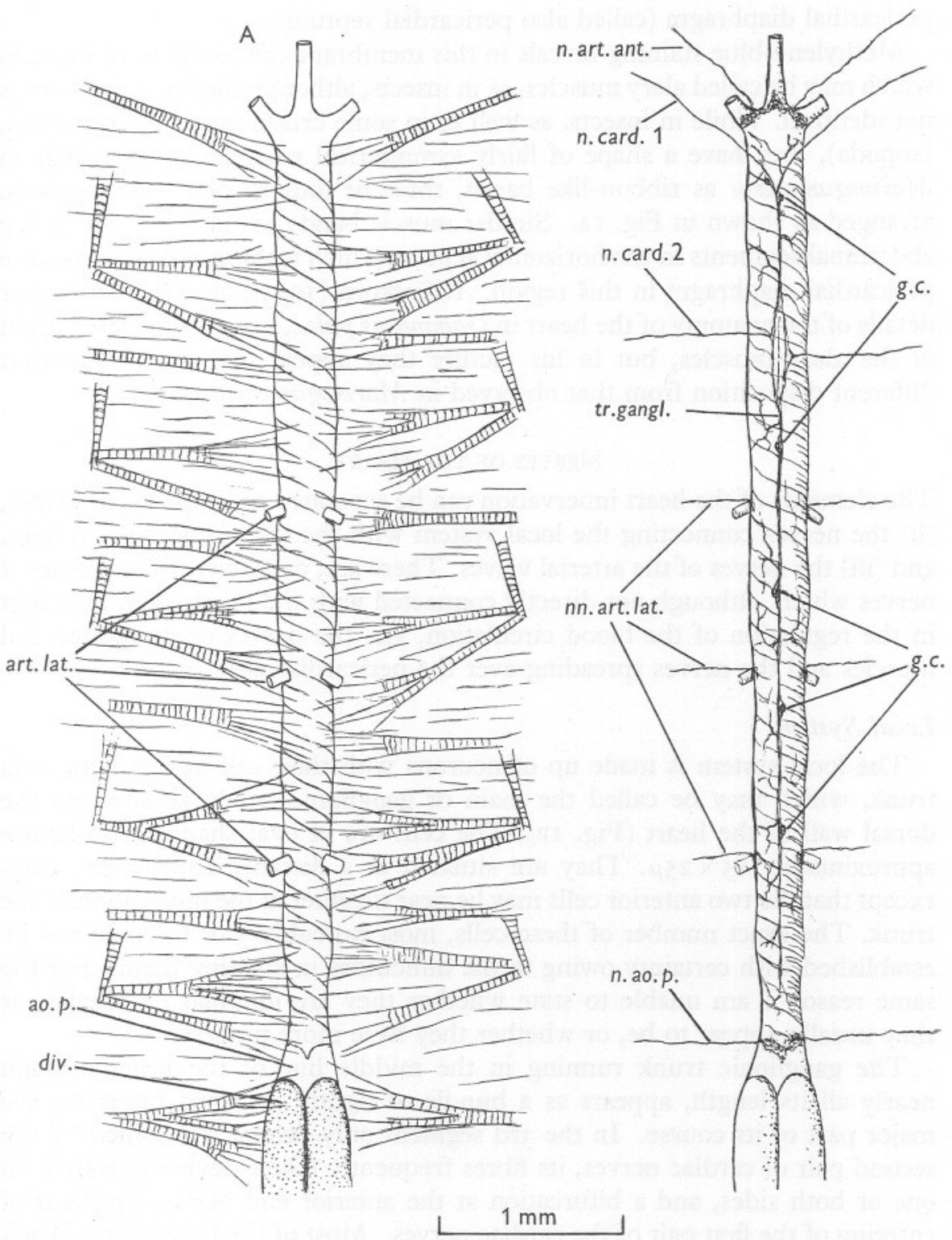

Fig. I. Marinogammarus marinus. A: view of the heart from the ventral side with its arteries and alary muscles of the pericardium. art.lat. lateral arteries of the 4 th-6th thoracic segments; ao.p. posterior aorta; div. coecal diverticula. B: view of the heart from the dorsal side. tr.gangl. ganglionic trunk; g.c. nerve cells; n.card.I, first cardiac nerve; n.card.2, second cardiac nerve; n.art.ant. nerve of arterial valves at the anterior end of the heart; nn.art.lat. nerves of the valves of the lateral arteries; n.ao.p. nerve of the valve of the posterior aorta. The ostia, situated on the lateral wall of the heart, cannot be seen in both figures. 
side of the heart. The ventral side of the heart lies on and is connected with the pericardial diaphragm (called also pericardial septum).

Methylene-blue staining reveals in this membrane the presence of muscles which may be called alary muscles, as in insects, although their arrangement is not identical. While in insects, as well as in some crustaceans (Stomatopoda, Isopoda), they have a shape of fairly symmetrical triangles, they appear in Marinogammarus as ribbon-like bands, three or four in each half segment, arranged as shown in Fig. IA. Similar muscle bands are also present in the abdominal segments in the horizontal septum which is the continuation of the pericardial diaphragm in this region. Klövekorn (I934), among many other details of the anatomy of the heart in Gammarus pulex, gives also a description of the alary muscles, but in his picture they appear to have a somewhat different disposition from that observed in Marinogammarus.

\section{Nerves of the Heart}

The elements of the heart innervation can be separated as (i) the local system, (ii) the nerves connecting the local system with the central nervous system, and (iii) the nerves of the arterial valves. There are, moreover, two systems of nerves which, although not directly connected with the heart itself, take part in the regulation of the blood circulation, viz. the nerves of the pericardial muscles and the nerves spreading over the pericardial wall.

\section{Local System}

The local system is made up of neurons with their cell bodies lying in a trunk, which may be called the main or ganglionic trunk, situated on the dorsal wall of the heart (Fig. IB). The cells are of oval shape and measure approximately $15 \times 25 \mu$. They are situated at a distance from each other, except that the two anterior cells may lie near together at the bifurcation of the trunk. The exact number of these cells, most probably 5 or 6 , could not be established with certainty owing to the difficulties in staining them. For the same reason I am unable to state whether they are unipolar or bipolar, as they usually appear to be, or whether they have more processes.

The ganglionic trunk running in the middle line of the heart through nearly all its length, appears as a bundle of tightly collected fibres for the major part of its course. In the 3 rd segment only, where it is joined by the second pair of cardiac nerves, its fibres frequently take a deviating course on one or both sides, and a bifurcation at the anterior end marks the point of entering of the first pair of the cardiac nerves. Most of the fibres of the trunk are the axons of its own nerve cells. They give off branches which, with their further divisions, supply the muscle fibres of the heart including those of the ostia but not those of the arterial valves. The finer ramifications are difficult to stain, but whenever the reaction is more favourable they appear, as in other crustaceans, abundantly distributed among the muscle fibres. 


\section{Nerves Connecting the Local System with the Central Nervous System}

Two paired nerves which may be called the first and second cardiac nerves run from the neural cord to the ganglionic trunk of the heart (Fig. IB, n.card. I, n.card. 2). The fibres of the first of them are conveyed by a trunk emerging from the posterior part of the infraoesophageal ganglion (Fig. 2, n.card. I). Not far from its origin it crosses a nerve trunk arising more anteriorly from the same ganglion and carrying fibres to the pericardial muscles and pericardial organs, and associates with it for a short distance (Fig. 2, $X$ ). As, besides, there are in this region anastomoses between various nerves, it cannot be excluded that in the cardiac nerve fibres of some other origin may also be present. From the point of junction mentioned above this nerve takes a more superficial course crossing the extensor muscles on their outer side near to their insertions. It then crosses on its inner side a thin oblique muscle (Fig. 2, $m$ ), inserting into the integument above the anterior end of the heart, and gives off a branch spreading over this muscle in a dense plexus. Passing on to the dorsal side of the heart the Ist cardiac nerve forms a Y-shaped figure with its fellow from the opposite side and with the ganglionic trunk.

The second cardiac nerve (Fig. 2, n.card. 2) runs to the heart with the dorsal branch of the segmental nerve originating from the connective linking the Ist and 2nd free thoracic ganglia (Fig. 2, n.segm.).This branch is a mixed nerve containing motor fibres passing to the dorsal layer of the extensor muscles, sensory fibres coming from the integument, fibres of the muscle receptors, and fibres to the pericardial organs (Fig. 2, on the right). The nerve running to the heart separates from other fibres at the lateral border of the median portion of the extensor muscles and passes on to the dorsal heart surface. It enters, often asymmetrically to that of the opposite side, the ganglionic trunk or one of its diverging branches, and bifurcates into branches running into opposite directions (Fig. IB).

Each of the two cardiac nerves appears to consist of more than one fibre, but it is very difficult to ascertain their true number. Neither has it been possible to trace their course in the ganglionic trunk for a longer distance and find out their terminations. It may be assumed, with a great degree of probability, that they enter into relation with the cells of the local system.

\section{Nerves of the Arterial Valves (Fig. IB)}

The nerves supplying the muscles of the arterial valves have no connexion with the elements of the systems described above. Two pairs of these valve nerves, viz. running to the anterior and posterior end of the heart, can be more easily noticed. The first of them approach the heart obliquely from the sides and, to judge from the direction of their course, come from the infraoesophageal ganglion, but could not be traced up to their origin. On reaching the valves the nerve fibres break up in a characteristic pattern of ramifications 


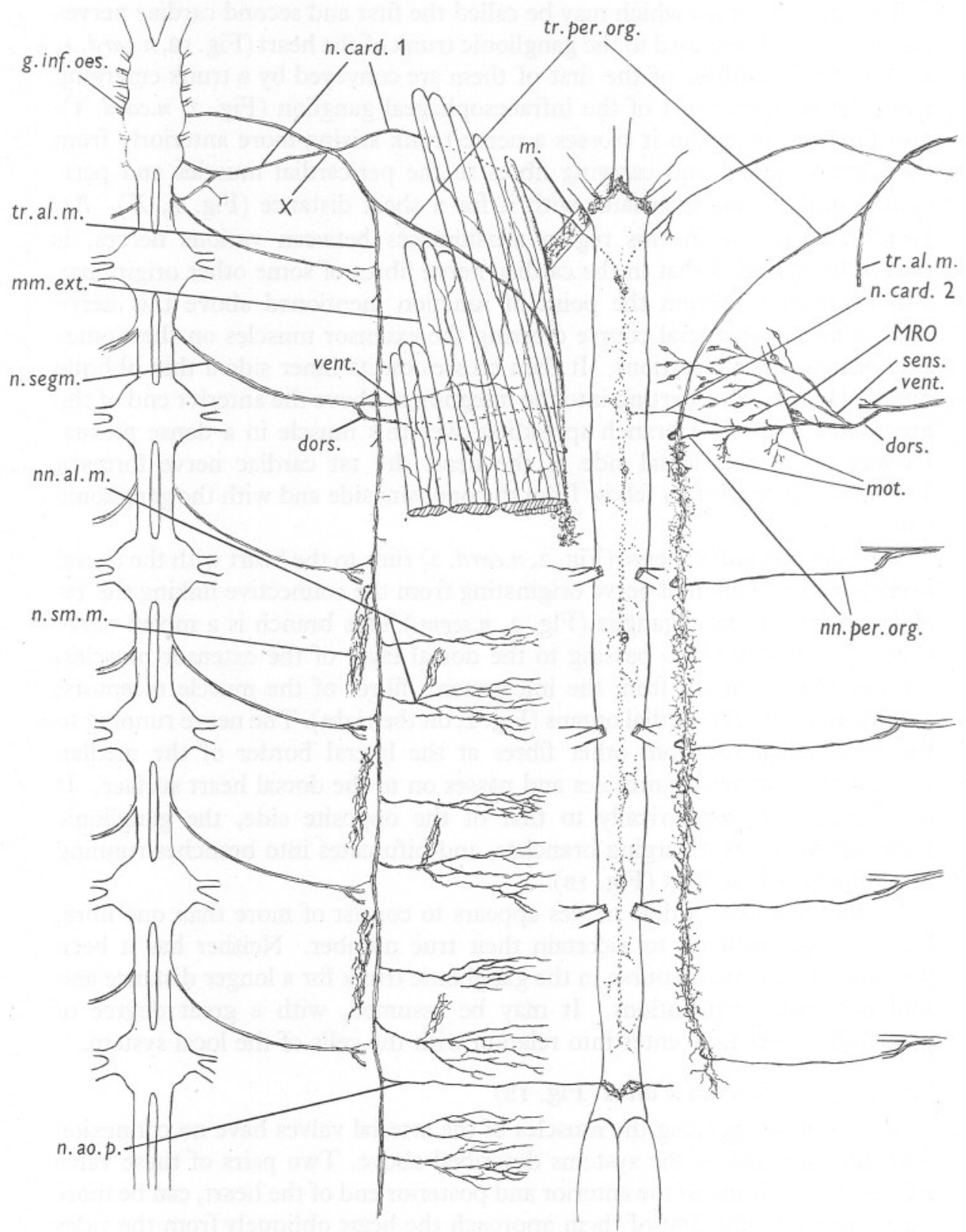

Fig. 2. See opposite page. 
different from that observed on the heart muscles. Some branches pass on to the valve of the anterior aorta and probably are the only source of innervation of this valve, since no separate nerve could be noticed to run down the aorta.

The nerves of the valve of the posterior aorta run transversely on the ventral surface of the extensor muscles. They arise from the longitudinal trunk giving off fibres supplying the alary muscles of the pericardium (Fig. 2, n.ao.p.), but, as pointed out below, this does not necessarily imply that they have a common origin and are in functional relation with this system of nerves.

The nerves of the valves of the arteries in the 4 th-6th segments are not easily noticeable. Tiny filaments of transverse direction pass on to these valves, but do not stain clearly. Moreover, very fine connective tissue fibres running also transversely in the pericardial septum and looking often very similar to the fine nerves, contribute to the difficulties of interpretation.

\section{Nerves of the Alary Muscles}

The nerves of the alary muscles of the pericardium are given off by a trunk running on each side through the thorax and the abdomen at the lateral edge of the extensor muscles (Fig. 2, tr.al.m.). This trunk takes origin in the infraoesophageal ganglion, being in the first part of its course united into a common bundle with the trunk of the pericardial organs, as well as with some fibres supplying the ordinary muscles. When crossing the successive segmental nerves the trunk of the alary muscles is joined by fibres branching from these nerves, but whether these additional fibres take part in the innervation of the alary muscles, or travel in the trunk for a short distance only to leave it and supply the ordinary muscles, I cannot say. The branches innervating the alary muscles (Fig. 2, nn.al.m.) are of very fine calibre. There seem to be more

Fig. 2. Marinogammarus marinus. Nerve elements controlling the blood circulation as seen in an animal sectioned along the left side and opened so that the neural cord is viewed from the dorsal side and the heart from its ventral side (semi-diagrammatic). The nerves represented in one plane of the drawing are situated in the animal in various planes and those running transversely follow in their course the curvature of the body; the nerves of the dorsal heart wall, same as in Fig. I B, but seen here by transparency are drawn in dotted lines. mm.ext. extensor muscles sectioned at the level of the 3 rd thoracic segment (in numbering the segments the first of thoracic segments is considered as fused with the cephalothorax);.m. small oblique muscle; g.inf.oes. infraoesophageal ganglion; n.card. I, Ist cardiac nerve; $X$, anastomose between the first cardiac nerve and the trunk bifurcating into the trunk of the alary muscles (tr.al.m.) and the trunk of the pericardial organs (tr.per.org.); n.segm. nerve arising from the connective and running to the dorsal part of the 3 rd thoracic segment; vent., dors., its ventral and dorsal branches; on the left side of the figure the ventral branch is seen ramifying on the ventral side of the extensor muscles; on the right side the dorsal branch is shown conveying: sensory nerves (sens.), motor nerves to the superficial layer of the extensor muscles (mot.), second cardiac nerve (n.card. 2), nerve to the pericardial organs (nn.per.org.), and nerves of the muscle receptor organs (MRO); nn.al.m. nerves of the alary muscles; n.sm.m. nerve of small muscles at the lateral edge of the pericardial diaphragm; n.ao.p. nerve of the valve of the posterior aorta. 
than one of them in each half segment, but they do not stain well and even in good preparations are difficult to detect.

From the same trunk arise also branches supplying small segmentally arranged muscles lying close to the trunk (Fig. 2, n.sm.m.). These muscle slips of longitudinal and slightly oblique direction are situated at the lateral edge of the extensor muscles, but appear as independent muscle units. As pointed out below, they may possibly be in some functional relation to the pericardial septum.

\section{Nerves of the Pericardial Wall (Pericardial Organs)}

The nerve trunk of the pericardial organs, after separating from the trunk of the alary muscles, crosses the ventral surface of the extensor muscles obliquely, and in the region of the 3 rd thoracic segment passes on to their median surface (Fig. 2, tr.per.org.). In this position it runs backwards as far as the 7 th segment. As the median surface of the extensor muscles constitutes the lateral wall of the pericardial cavity, this trunk comes to lie on the pericardial wall for most of its course. During this course it becomes strengthened by fine fibres which arise from the dorsal branches of the segmental nerves and curve ventrally round the convexity of the extensor muscles to join the pericardial trunks (Fig. 2, nn.per.org.). The first of them is situated in the 3rd segment and that can be more easily noticed. Their probable number is five on each side, but owing to their situation, particularly unfavourable both for staining and observation, this number is not certain.

There is a clear difference in structure of the trunks of the pericardial organs and that of the ordinary nerves, and even between the portions of the pericardial trunks themselves. Before passing on to the pericardial wall they look quite like the ordinary nerves, and so do the branches coming from the segmental nerves. On the pericardial wall, however, the trunks become broader, show uneven outlines, and look as if they consist of very small granules. Closer scrutiny of the best preparations reveals that the nerve fibres here break up into very fine filaments and during the staining process these in turn disintegrate into granules. In most favourable cases strands of such fine neuropile-like nerve tissue may be seen branching from the main trunks and entangling in a plexus spreading over the lateral wall of the pericardium. The more frequently observed uneven appearance of the trunk outlines is due to the incomplete staining of the strands starting from the trunks. Occasionally some bodies can be noticed which might be interpreted as indistinctly stained nerve cells, but their nature has always been doubtful.

Owing to the size of the animals and to technical difficulties in obtaining nerve preparations the picture of the nerves in the pericardium is not so impressive as in large crustaceans, but there can hardly be any doubt that these elements belong to the same category as the pericardial organs of the Stomatopoda and Decapoda described previously (Alexandrowicz, I953 $a, b$ ). 


\section{Discussion}

The innervation of the heart of Marinogammarus proves to have much in common with that of other Crustacea and in particular, owing to the shape and anatomy of the heart, with that of the Isopoda (Alexandrowicz, I952). There is, however, a difference in the situation of the ganglionic trunk, in that in the Isopoda investigated (Ligia oceanica, Mesidotea entomon, Idotea emarginata) it lies on the inner surface of the heart, while in the Gammaridae it lies outside of it. The latter position is the same as in the Stomatopoda (Alexandrowicz, I934), and, according to some observations I have recently made, in the Mysidacea too. Otherwise the ganglionic trunk appears to have a similar shape and structure as in isopods, i.e. it is composed of a few neurons of the local system and some fibres connecting this system with the neural cord. As in other crustaceans the local system should be regarded as an autonomous apparatus ruling the heart beat.

The situation of the heart in the Amphipoda extending up to the cephalothorax proved to be more favourable than in the Isopoda for the observation of the nerves connecting the heart with the neural cord, and gave the chance of establishing the origin of these nerves as from the infraoesophageal ganglion and the Ist free thoracic ganglion. There are, however, still some doubtful points. For instance, as the Ist thoracic segment is fused with the cephalothorax and its ganglion with the infraoesophageal ganglionic mass, the question remains whether the Ist cardiac nerve originates in the ganglion of the Ist thoracic segment, which judging from its course is more likely, or from one of the cephalic segments. If the former is true, the cardiac nerves, which presumably have a regulatory function on the heart rhythm, would originate in the two anterior thoracic segments (the term segment being used as equivalent to metameres).

As regards the innervation of the arterial valves a particular feature in gammarids would be the absence of the nerve running down the anterior aorta, which, known as nerve of Lemoine, was one time considered to be the main nerve of the heart of decapods, but was found in these animals as well as in stomatopods and isopods to supply only the valve of the anterior aorta (Alexandrowicz, 1932, 1934, I952). In gammarids, unless this negative evidence is due to some particularly refractory behaviour of this nerve to staining with methylene blue, it appears to be wanting.

The question whether the innervation of the arterial valves is linked with that of the pericardial muscles in a way securing their synchronous action remains open. In decapods all these nerves, with exception of that supplying the valve of the anterior aorta, arise from a common bundle, whereas in stomatopods the two systems have independent courses; in isopods their relations have not been determined, and the fact that in Marinogammarus the nerve to the posterior aorta arises from the trunk of the alary muscles does not prove that the fibres of both have a common origin. 
The assumption that the nerves of the valves and of the alary muscles are in functional relation is insecure until the role of the pericardial muscles is definitely established. The opinions of the writers who have studied this problem in crustaceans as well as in insects differ as to whether the function of these muscles may be passive or active, i.e. whether they simply strengthen the elastic resistance of the pericardial septum, or whether their contractions contribute to the movements of this septum. Even the two authors who described the alary muscles in amphipods, v. Haffner (1934) in Phronima sedentaria, and Klövekorn (1934) in Gammarus pulex, are not quite in accord. On this question the following remarks may be added. First, it seems rather unlikely that organs made up of cross-striated muscle tissue and supplied by a special system of nerves would function merely as elastic bands; and, secondly, that considering their possible action there should be taken into account not only their relation to the heart-beat, but their possible coordination with the movements of the animal. It is obvious that during the movements of the body the respective pressure in the large sinuses can vary widely and if, as is likely, such fluctuations of the blood pressure in the pericardial cavity would impair the heart action, the appropriate contraction of the alary muscles might regulate the position of the pericardial diaphragm and compensate for these changes. It is, moreover, possible that not only the alary muscles, but also those small muscle slips mentioned above as receiving their nerve supply from the trunk of the alary muscles, are active in the same way. They end, in fact, exactly at the level of the lateral attachment of the pericardial septum and it appears very likely that their contractions may have a pulling action on the latter. It should be mentioned that in Phronima v. Haffner has found small muscles attached to the lateral edge of the pericardium which, as he puts it, 'dienen...zur Befestigung des Pericardialseptums'.

The nerves expanding over the pericardial wall in neuropile-like plexuses show such a resemblance in their mode of termination to those found in stomatopods and decapods that their inclusion in that category of elements called pericardial organs may be considered as well founded. It is therefore highly probable that they have a neurosecretory function, releasing into pericardial cavity some hormone or hormones which influence the heart rhythm in a similar way as in those animals (Alexandrowicz \& Carlisle, I953). In the present description of these organs it has been pointed out that no uncontestable evidence of the presence of nerve cells in them could be obtained. If the assumption that they are missing is correct, all the fibres of the pericardial trunks would come from the cells situated in the central nervous system, and thus this arrangement in gammarids would resemble more that in decapods than in stomatopods, since in the latter most of the fibres of the pericardial organs originate in the nerve cells lying outside the ganglionic cord.

It is interesting to note that different elements of the systems described above, viz. the first of the cardiac nerves, the trunk of the alary muscles, and 
the trunk of the pericardial organs, originate in the infraoesophageal ganglion. Hence it may be conjectured that the centre controlling the blood circulation could be localized in this part of the nervous system. Admittedly some of the nerves described, as the and cardiac nerve and the additional nerves to the pericardial organs, start from the thoracic ganglia, but it is possible that the cell-bodies of their neurons are situated in the infraoesophageal ganglion while their axons run down the neural cord to leave it with the nerves arising farther backwards. This problem must await solution until by successful tracing of all the nerves in question their true origin and the connexions of their neurons can be established.

\section{SUMMARY}

In the heart of Marinogammarus marinus three systems of nerve elements have been found, viz. (I) a local system consisting of a ganglionic trunk situated on the dorsal surface of the heart and sending branches to the muscle fibres, (2) two paired nerves, termed the Ist and 2nd cardiac nerves, connecting the local system with the central nervous system, and (3) nerves supplying the muscles of the arterial valves. It is assumed that the local system rules the beat of the heart and that the cardiac nerves have a regulating function.

Not directly connected with the heart, but having relation to the blood circulation are two sets of nerves: (I) nerves supplying the alary muscles, presumably regulating the pressure in the pericardial cavity, and (2) nerves spreading over the lateral wall of the pericardium in neuropile-like plexuses and supposed to have a neurosecretory function.

It is suggested that the centre controlling the blood circulation could be localized in the infraoesophageal ganglion.

\section{REFERENCES}

Alexandrowicz, J. S., I932. The innervation of the heart of the Crustacea. I. Decapoda. Quart. F. micr. Sci., Vol. 75, pp. 18I-249.

1934. The innervation of the heart of the Crustacea. II. Stomatopoda. Quart. 7. micr. Sci., Vol. 76, pp. 51 I-48.

- 1952. Innervation of the heart of Ligia oceanica. F. Mar. biol. Ass. U.K., Vol. 3I, pp. $85-96$.

- I953a. Nervous organs in the pericardial cavity of the decapod Crustacea. F. Mar. biol. Ass. U.K., Vol. 31, pp. 563-80.

- I $953 b$. Notes on the nervous system in the Stomatopoda. II. The system of dorsal trunks. Pubbl. Staz. zool. Napoli, Vol. 24, pp. 29-39.

Alexandrowicz, J. S. \& CARLisle, D. B., I953. Some experiments on the function of the pericardial organs in Crustacea. F. Mar. biol. Ass. U.K., Vol. 32, pp. 175-92.

v. HAFFNER, K., I934. Der Blutkreislauf von Phronima sedentaria, mit besonderer Berücksichtigung des lacunären Systems. Z. wiss. Zool., Bd. I46, pp. 283-328.

KlöveKoRn, J., I934. Das Organsystem der Blutbewegung bei Gammarus pulex L. Z. wiss. Zool., Bd. 146, pp. 153-92.

Sexton, E. W. \& Spooner, G. M., I940. An account on Marinogammarus (Schellenberg) gen.nov. (Amphipoda), with a description of a new species, M. pirloti. F. Mar. biol. Ass. U.K., Vol. 24, pp. 633-82. 